\begin{tabular}{|l|l|l|l|l|l|} 
Revista Duazary & ISSN: 1794-5992 & Vol. 12 & No. 1 & $49-58$ & Enero - Diciembre de 2015 \\
\hline
\end{tabular}

\title{
LA DISCAPACIDAD Y SU ESTADO ACTUAL EN LA LEGISLACION COLOMBIANA
}

\section{DISABILITY AND CURRENT STATUS IN THE COLOMBIAN LEGISLATION}

\author{
TITULO CORTO: LA DISCAPACIDAD Y SU ESTADO ACTUAL
}

Ana Milena Martínez-Rozoํㅜ Ana Fernanda Uribe-Rodríguez², Héctor José Velázquez-González ${ }^{3}$

Recibido en Octubre 30 de 2014

Aceptado en Diciembre 15 de 2014

\section{RESUMEN}

Conocer el estado actual de la legislación Colombiana frente a cinco ámbitos vitales que afrontan las personas con discapacidad: salud, educación, empleo, transporte y accesibilidad; realizando una comparación entre dicha legislación con los resultados encontrados en la encuesta de calidad de vida realizada en el 2012 por el Departamento Administrativo Nacional de Encuestas DANE y el Informe inicial sobre la Convención de los Derechos de las Personas con Discapacidad, se encontró que, aunque existe legislación a favor de los derechos de las personas con discapacidad, el goce efectivo de la salud, la educación, el empleo, el transporte y la accesibilidad es mínimo. Es una población que presenta analfabetismo, un alto porcentaje no se encuentra afiliado al sistema de salud y en el mercado laboral las oportunidades para esta población son escasas. Con respecto al transporte y accesibilidad, entendida esta última como señalización, movilidad y comunicación, aún se encuentran barreras arquitectónicas. Se requiere que el país avance en el cumplimiento de los derechos de las personas con discapacidad, para evitar toda clase de discriminación y marginación garantizando una mejor calidad de vida para todos y se dé un empoderamiento mayor a las personas con discapacidad y la comunidad en general.

Palabras Clave: Legislación; Discapacidad; Salud; Educación; Empleo; Accesibilidad (fuente: DeCS)

\section{Aвstract}

The main objective of this paper is to explore the current status of the Colombian legislation against five vital areas faced by people with disabilities: health, education, employment, transportation and accessibility; making a comparison between this legislation with the results found in the Quality of Life Survey conducted in 2012 by theNational Bureau of Surveys DANE and the Initial Report on the Convention on the Rights of Persons with Disabilities. The results found that although there is legislation in favor of the rights of persons with disabilities the full enjoyment of health, education, employment, transportation and accessibility is minimal. It is a population with illiteracy, a high

1. Psicóloga Joven investigadora Universidad Pontificia Bolivariana Bucaramanga-Colciencias, Colombia. Correo: anamilena.martinez@upb.edu.c

2. Doctora en psicología clínica y de la salud, docente asociada Universidad Pontifica Bolivariana Bucaramanga, Colombia. Correo: anafernanda.uribe@upb.edu.co

3. Estudiante de doctorado en psicología clínica, Pontificia Universidad Católica de Puerto Rico, Puerto Rico Correo: hectorvelazquez@pucpr.edu 
percentageis not affiliated to a health system and the labor market opportunities for this population are limited. With regard to transportation and accessibility, understood as signaling, mobility and communication, are still architectural barriers. Is required more advances in fulfilling the rights of the people with disabilities, to avoid all forms of discrimination and marginalization by ensuring a better quality of life for all. Also to promote empowerment of people with disabilities and the general community.

Keywords: Legislation; Disability; Health; Education; Employment; Accessibility (Source: DeCS)

\section{INTRODUCCIÓN}

A unque la discapacidad no es un problema de salud pública, sí debe ser considerada una prioridad para los diferentes países; principalmente por el incremento de la misma y los condicionantes sociales y ambientales que afecta a la persona con discapacidad y a su entorno ${ }^{1}$. De igual forma, una de las principales causales de discapacidad son las enfermedades generales y crónicas, y los accidentes públicos y laborales, como la actitud negativa discriminadora frente a la situación de discapacidad.

A nivel mundial el número de personas con algún tipo de discapacidad es mayor a mil millones, es decir, un 15\% de la población mundial; cifra que tiende a aumentar por el incremento de las enfermedades crónicas y el envejecimiento ${ }^{2}$. En Colombia, la población total de personas con discapacidad se encuentra en alrededor de 2.149.710, correspondiente al 4,7\% de la población según el DANE 3 ; por otra parte, el grupo de edad más afectado es el de las personas mayores de 60 años, representando un $47 \%$ de la población. La mayor causa de discapacidad reportada en el Sistema Integral de Información de la Protección Social (SISPRO) ${ }^{4}$, para el 2011, es la enfermedad general $(44,78 \%)$, seguida de la accidentalidad $(17,28 \%)$ y la alteración genética y hereditaria (15,09\%). La alteración predominante es la permanente del cuerpo, manos, brazos y piernas; que afecta a 543,213 personas según el Registro de localización y caracterización de personas con discapacidad-RLCPD ${ }^{4}$.

50

Las estadísticas mencionadas permiten comprender que la discapacidad se ha convertido en un tema de pertinencia académica e investigativa; $y$ a pesar de que su existencia es tan antigua como el hombre mismo, el interés y la importancia por dicha temática no se había despertado en las diferentes ramas de las ciencias humanas y en entes gubernamentales, como ha sucedido en pleno siglo $\mathrm{XXI}^{2}$. Precisamente, la comprensión del fenómeno sigue en proceso y la población discapacitada sigue sujeta a rechazo y discriminación en determinados contextos sociopolíticos y culturales ${ }^{5}$.

\section{MATERIALES Y MÉTODOS}

La investigación tuvo un enfoque cualitativo, basado en el análisis documental específicamente de legislación en discapacidad. Se hizo una búsqueda en bases bibliográficas como EBSCON, Redalyc, Scielo, Dialnet, Scopus. Utilizando como palabras claves para la búsqueda: Legislación, discapacidad, políticas públicas en discapacidad, educación, inclusión laboral, accesibilidad, normativas en salud, transporte incluyente, Colombia y la discapacidad.

Durante la investigación se establecieron cuatro fases. En primera instancia se realizó la recolección de datos bajo la técnica de rastreo documental en bases de datos. En un segundo momento, se clasificó la literatura según las variables de estudio. En un tercer paso, se realizó lectura de los documentos para la extracción de la información pertinente. Por último, en la cuarta fase, se ordenó la información encontrada para la estructurara del apartado de resultados. Se encontraron inicialmente cincuenta y ocho normas de políticas públicas en la legislación colombiana: veintiún normas en salud, trece en educación, doce en empleo, cinco en comunicaciones e información, dos en transporte, dos en recreación y deporte, dos en cultura y una en accesibilidad. También, se encontraron treinta artículos científicos que desarrollaban temáticas de acuerdo con las variables estudiadas. 


\section{RESULTADOS}

\section{Breve recorrido histórico del concepto de discapacidad en la legislación colombiana}

En Colombia la discapacidad comenzó a ser visible en la década de los cincuenta, con la aparición del Institución Nacional para Ciegos (INCI), el Instituto Nacional para Sordos (INSOR) ${ }^{6}$ y el instituto Roosevelt, pionero de la rehabilitación en Colombia²; pero no fue sino hasta 1981 cuando la legislación colombiana emitió el Decreto 2358 con el cual nació el Sistema Nacional de Rehabilitación? De igual forma, el Ministerio de la Salud (1985), creó la Resolución 14861 en la cual "se dictan normas para la protección, seguridad, salud y bienestar de las personas en el ambiente y en especial de los minusválidos"8. En este año surgió un término "minusválidos" que caracterizaba a las personas con la condición de discapacidad pero sin definición. Por tanto, se optó por la definición de la Organización de las Naciones Unidas (ONU) sobre minusválida entendida como "una situación de desventaja para un individuo determinado, de una deficiencia o de una discapacidad, que limita el desarrollo de un rol que es normal en su caso, en función de la edad, sexo y factores culturales y sociales" ${ }^{\prime}$. Esto contribuyó al esfuerzo realizado a nivel mundial desde hacía varios años para visualizar la discapacidad como tema relevante a nivel social y politico ${ }^{10}$.

En 1987 el Congreso de Colombia implementó la Ley 12, la cual estableció requisitos en cuanto a construcción y espacio público para la eliminación de barreras arquitectónicas, y la facilidad en el acceso de las personas con discapacidad. De igual forma, en esta Ley se empleó el término "personas con discapacidades" ${ }^{11}$, sin definirlo; por lo que se recurrió nuevamente a la definición utilizada por la ONU, la cual indicaba que una persona con discapacidad es aquella con "restricción o falta (debidas a una deficiencia) de la capacidad para realizar una actividad en la forma o dentro del margen que se consideran normales para un ser humano"'.

Para 1988, se creó la Ley 82, con el fin de "aprobar el convenio 159 sobre la readaptación profesional y el empleo de personas inválidas, adoptado por la conferencia general de la Organización Internacional del Trabajo (OIT) en su 69a , reunión en Ginebra en 1983"12, donde se define la persona inválida como "toda persona cuyas posibilidades de obtener y conservar un empleo adecuado y de progresar en el mismo queden sustancialmente reducidas a causa de una deficiencia de carácter físico o mental debidamente reconocida"12. Es evidente, la nueva perspectiva de las implicaciones de la discapacidad en el contexto laboral, siendo un tema clave en la inclusión. Dando un salto en la historia, en década de los 90 no se establecen o disponen leyes en la legislación colombiana que definiera el concepto de discapacidad. Solo hasta el 2002, en la Ley 769, se expide el Código Nacional de Tránsito Terrestre para el cumplimiento legal de sus disposiciones, y se define que un discapacitado es: "aquella persona que tiene disminuida alguna de sus capacidades físicas o mentales"13. Siendo esta la primera aproximación conceptual a la discapacidad. Sin embargo, solo hasta el 2007, con la Ley 1145, que se organiza el Sistema Nacional de Discapacidad y en ella se define que una persona con discapacidad es "aquella que tiene limitaciones o deficiencias en su actividad cotidiana y restricciones en la participación social por causa de una condición de salud, o de barreras físicas, ambientales, culturales, sociales y del entorno cotidiano"14

Concluyendo con el recorrido histórico de conceptos y definiciones, la Ley Estatutaria 1618 del 2013, brinda un concepto actual de "personas con y/o en situación de discapacidad”, las cuales define como:

"aquellas personas que tengan deficiencias físicas, mentales, intelectuales o sensoriales a mediano y largo plazo que, al interactuar con diversas barreras incluyendo las actitudinales, puedan impedir su participación plena y efectiva en la sociedad, en igualdad de condiciones con lo demás"15.

Es pertinente aclarar que una Ley estatutaria es de mayor relevancia que las Leyes ordinarias, debido a que la temática a tratar es de gran prioridad y su cumplimiento debe ser inmediato ${ }^{16}$. Dicha Ley surgió de la necesidad de prestar más atención al cumplimiento de sus derechos y disminuir el índice de toda clase de discriminación frente a esta población. Esta Ley es la norma vigente en la legislación colombiana que abarca de una manera global la discapacidad con la finalidad de proteger en todos los ámbitos los derechos de las personas con discapacidad. En cuanto al desarrollo histórico del concepto de discapacidad, este ha evolucionado en la medida que se han dado aproximaciones a la comprensión de la discapacidad como un fenómeno social, individual y de pertinencia política; y a su vez, por los avances científicos obtenidos en la evaluación, intervención y manejo de las personas con discapacidad. El tener un concepto claro, amplio e incluyente de las personas con discapacidad influye en todos los aspectos ético-científicos de la visión 
integral de la misma y sus implicaciones tanto para las personas como para la sociedad.

\section{Esfuerzos legislativos en pro de la población con discapacidad}

La construcción legislativa sobre discapacidad implica reflexiones y planteamientos de diferentes sectores en los cuales están implicados las personas con discapacidad. Es relevante mencionar, que aunque se cuenta con legislación pertinente y vigente, el seguimiento y cumplimiento de la misma presenta dificultades importantes ${ }^{17,5}$. Teniendo en cuenta lo anterior se realiza una revisión y reflexión de la legislación para personas con discapacidad en el contexto colombiano; específicamente en las políticas y normas más relevantes en temas de salud, educación, empleo, transporte y accesibilidad que salvaguardan los derechos de la población colombiana con discapacidad.

\section{Legislación en Salud}

La Constitución Política de 1991, la Ley $60^{18}$ de 1993 y la Ley $100^{19}$ del mismo año aportaron en la reforma al Sistema de Salud en Colombia; principalmente en lo relacionado con lograr un servicio de salud más solidario y equitativo entre todos los ciudadanos, y que los que tienen mayores ingresos, subsidien a los menos favorecidos ${ }^{20}$. A pesar de esto, persisten las falencias en la vinculación total de las personas con discapacidad al Sistema de Salud en Colombia. Según los resultados encontrados en la Encuesta Nacional de Calidad de Vida realizada en el 2012 por el DANE³ a la fecha, en Colombia había 46.168. 649 habitantes, de los cuales 2.149 .710 presentaban alguna discapacidad. En cuanto a la vinculación a la salud 1.989. 266 , poseen afiliación a la salud, donde más de la mitad $(57,7 \%)$, corresponden al régimen subsidiado y un 24 , $4 \%$ no reporta ningún tipo de afiliación en salud. ${ }^{3}$

Aunque se establece que "los Estados deben asegurar la prestación de atención médica eficaz para las personas con discapacidad" ${ }^{21}$, en Colombia, no se tiene una cobertura en el Sistema de Salud para todas las personas con discapacidad. Por otra parte, las personas con discapacidad que se encuentran afiliadas al sistema de salud deben de recurrir a la acción de tutela para hacer valer en totalidad el cumplimiento de sus derechos a la salud ${ }^{22}$; debido a los altos costos que estos demandan en procesos de rehabilitación, intervenciones tempranas, vigilancia de condiciones crónicas y el uso de tecnología ${ }^{23}$. En el informe inicial sobre la implementación de la Convención sobre los Derechos de las Personas con
Discapacidad en Colombia ${ }^{24}$, entregado a la ONU en el año 2013, se manifiesta que actualmente se están tomando medidas legislativas para reglamentar el Artículo 66 de la Ley 1438 de 2011; por medio de la cual, se ordena una atención integral en salud a personas con discapacidad ${ }^{25}$. Por otra parte, hacen mención de que presentan limitaciones en la inclusión de nuevas tecnologías al Plan Obligatorio de Salud, ya que éstas se encuentran sujetas a costos, calidad y efectividad. Estos aspectos implican que la atención en salud de las personas con discapacidad no sea integral como lo exige la norma; tampoco se han implementado en su totalidad rutas de acceso para la atención en las instituciones de salud, y por último, se han trazado planes de desarrollo en salud pública a largo plazo, donde se necesitan intervenciones inmediatas ante una realidad inminente por el incremento de la discapacidad.

Desde el quehacer propio del psicólogo, el abordaje de la persona con alguna discapacidad debe ir precedida de su reconocimiento como persona, yendo así más allá de la sola asignación de una etiqueta con valor nosológico. De esta manera se podrá reconocer en la persona con discapacidad no a un individuo con determinada condición diferenciadora, sino más bien a una persona con un patrón de posibilidades ante las exigencias de su medio próximo, es decir, una persona como cualquiera, poseedora de puntos fuertes o débiles según los condicionamientos ambientales ${ }^{26}$.

\section{Legislación en Educación}

La educación es reconocida como un derecho básico universal, soporte de la sociedad para adquirir un desarrollo a nivel de oportunidades y garantías para todos $^{27}$. En consecuencia, la atención educativa de las personas con discapacidad es una obligación del Estado, según la Constitución Política de Colombia de 199128, reglamentada en Leyes, decretos y resoluciones.

Tomando como referencia una de las últimas mediciones realizadas por el DANE, en la Encuesta de Calidad de Vida del año $2012^{3}$, se encontró que el $80 \%$ del total de personas de entre cinco y nueve años con alguna discapacidad no han alcanzado ningún nivel educativo; mientras que el $13,2 \%$ había cursado solo preescolar. En el grupo de edades de 10 a 17 años, el $47,1 \%$ de las personas con discapacidad no había aprobado ningún nivel educativo y el 33,9\% había aprobado básica primaria. Según esta misma fuente, en el grupo de entre 18 y 39 años de edad, $24,7 \%$ de los encuestados no había alcanzado ningún 
nivel educativo, mientras que la proporción de personas que alcanzó educación superior fue de 9,7\%. También se encontró que en las personas con discapacidad el porcentaje de analfabetismo fue de $24,1 \%$, mientras que en la población sin discapacidad fue de un 6,9\%.

Como se puede observar, los resultados indican que entre los diferentes rangos de edades de la población con discapacidad, los niveles educativo son bajos y el nivel de analfabetismo es más alto que en la población sin discapacidad. Con relación al nivel de estudios se puede indicar que las políticas y reglamentaciones educativas en Colombia se han restringido más al tipo de educación básica y primaria y no se ha hecho extensiva al nivel superior $^{22}$. Por lo cual, se ha negado la posibilidad a las personas con discapacidad de tener acceso a la educación y al desarrollo social ${ }^{29}$, a adquirir conocimientos de nivel superior, a prepararse para ingresar al mundo laboral y tener la oportunidad de contribuir a su desarrollo y al de una sociedad ${ }^{30}$. En consecuencia, se limita el potenciar aspectos de su personalidad, tales como la integración y desinstitucionalización exitosa, la aparición de problemas de comportamiento y psicológicos, y el prevenir el abuso sexual ${ }^{31}$.

Según el informe entregado a la ONU sobre la implementación de la Convención de los Derechos de las Personas con Discapacidad ${ }^{24}$, el gobierno nacional indica que se han adoptado medidas para asegurar que todos los niños y niñas con discapacidad tengan acceso a una educación temprana por medio de diferentes programas generados por el Ministerio de Educación Nacional, como una medida para dar cumplimiento a los compromisos asumidos en la convención. Ante esto y los resultados obtenidos por el DANE, es evidente que el acceso a la educación para las personas con discapacidad sigue siendo limitado; ya que el Estado cumple con crear los programas, pero no los lineamientos de acceso para que estos sean sostenibles en el tiempo, pues no todas las personas con discapacidad acceden a estos programas porque existen otros problemas de fondo como la accesibilidad a las instituciones, el traslado, contar con un acompañante, etc.

El profesional que se desempeñe en ámbitos educativos con esta población deberá propender por la promoción de seres humanos autónomos, eficaces y proactivos ${ }^{32}$. Asimismo, generar estrategias investigativas que permitan consolidar estrategias pedagógicas cada vez más eficaces para el trabajo con personas con discapacidad; a fin de que se vea asegurado no solo su legítimo derecho a la educación, sino a una educación de calidad que les permita desarrollar sus capacidades con miras a la realización personal y laboral dentro de su proyecto de vida $^{33,34}$.

\section{Legislación en Empleo}

Las personas con discapacidad presentan mayor probabilidad de estar desempleadas, y cuando obtienen un empleo por lo general tienen la percepción de recibir menores ingresos comparados con la población sin discapacidad $^{6}$. La Organización Mundial de la Salud (OMS) indica como uno de los principales derechos de las personas con discapacidad la inclusión laboral, la cual debe ser en igualdad de condiciones respecto a tareas, salarios y horarios con las demás personas contratadas en una empresa ${ }^{17}$. Lo cual permitiría contribuir en la calidad de vida de las personas con discapacidad fomentando el desarrollo "integral dentro de las diferentes esferas del desarrollo del ser humano"35. Pero la realidad es que diversos estudios coinciden en destacar las bajas tasas de inclusión laboral como la mayor dificultad de las personas con discapacidad en los países desarrollados ${ }^{36-38}$.

En Colombia la idea de vincular personas con discapacidad en las empresas surge de los grandes beneficios que éstas adquieren, como la disminución en el porcentaje tributario que la empresa debe pagar al Estado ${ }^{39}$. Desde el año 1997 el Congreso de la República de Colombia creó la Ley $361^{40}$, la cual ofrece beneficios tributarios a aquellas empresas que vinculen personas con discapacidad a su organización; estas empresas tendrán preferencias en los procesos de licitación de contratos, otorgamientos de créditos para el desarrollo de programas que impliquen la participación de personas con discapacidad y reducción de un porcentaje de hasta $200 \%$ en la renta por concepto de salarios y prestaciones sociales.

Comparando los resultados que se obtuvieron en la medición de Calidad de Vida en el 2012, con las Leyes y decretos existentes sobre inclusión laboral, se encontró que sólo un 28,6\% de las personas con discapacidad se encontraban ocupadas; respecto a la remuneración recibida se encontró que 30 de 100 personas con discapacidad recibe menos de $\$ 500.000$ mensuales, lo cual indica que el $91 \%$ de las personas perciben que reciben ingresos inferiores a un salario mínimo legal vigente $(\mathrm{SMLV})^{3}$. Lo anterior evidencia que aún se siguen teniendo estereotipos de rechazo y discriminación frente a esta población ${ }^{39}$. Cardona ${ }^{41}$ indica que el índice de inactividad es más alto en las personas con discapacidad 
y se encuentra relacionado con el aspecto físico, siendo un tipo de discriminación injustamente arraigado en el medio ${ }^{22}$.

Por otra parte, las medidas que ha adoptado el gobierno colombiano para generar inclusión de las personas con discapacidad en el campo laboral reportado en el informe de implementación de la Convención sobre los Derechos de las Personas con Discapacidad ${ }^{24}$, son convenios desde el sector público y privado; dando hincapié a programas de responsabilidad social empresarial, donde se encuentran vinculadas empresas que promueven la apertura de plazas productivas específicamente para personas con discapacidad. Aunque se ha generado conciencia y sensibilización a nivel empresarial sobre la inclusión laboral de personas con discapacidad, el índice de éste sigue siendo reducido, los empresarios desconocen los programas de inclusión laboral existentes y las estructuras arquitectónicas de las empresas siguen siendo inasequibles, la cual es una de las principales causas que impide la contratación de personas con discapacidad.

El profesional activo en el sector laboral y organizacional debe velar porque los procesos como la selección de personal para los distintos cargos, sean realizados en condiciones equitativas. De forma tal, que las personas con discapacidad no sean rechazadas por ello, sino que cuenten con posibilidades reales de acceder a las diversas oportunidades de trabajo $0^{42-44}$, ya que en la mayoría de las ocasiones la discapacidad es más un producto de la disparidad entre condición individual y oportunidades del ambiente inmediato ${ }^{45}$.

\section{Legislación en Transporte y Accesibilidad}

El derecho a la movilidad representa la inclusión más importante de acceso en las personas con discapacidad, ya que las barreras físicas y sociales limitan la autonomía afectando su independencia y dignidad ${ }^{22}$.

En Colombia las primeras normas en transporte y accesibilidad que benefician a las personas con discapacidad surgieron en el año 1987 con la Ley 12, ${ }^{46}$ donde se tomaron medidas para la construcción de 54 espacios públicos. Luego se tuvo el Decreto 1660 del año $2003^{47}$, el cual establece mecanismos de accesibilidad en los medios masivos de transporte; el Decreto 1538 del $2005^{48}$ que garantiza condiciones de accesibilidad en espacios públicos y vivienda; la Ley 1083 del $2006^{49}$ vela por la movilidad de las personas con discapacidad dentro del perímetro urbano;, la resolución 4659 del $2008^{50}$ asegura la movilidad en trasporte masivo urbano, municipal y distrital; y por último, la Ley 1287 de $2009^{51}$ que respalda el establecimiento de normas de accesibilidad en bahías de estacionamiento.

Es de notar que la mayoría de normas se limitan a la construcción de rampas, puertas de acceso amplio, bahías, pasamanos y acceso al transporte público incluyentes para las personas con discapacidad física. Pero hay que tener en cuenta que se encuentran limitaciones para los otros tipos de discapacidad ya que hace falta "la implementación del braille en avisos y comunicaciones, la implementación de información sonora, el uso de guías e intérpretes de la lengua de señas, entre otros que constituyen medidas humanas y de diseño para la accesibilidad",22 lo cual proporcionaría más autonomía en el diario vivir de las personas con discapacidad.

En el aspecto de transporte y accesibilidad, en el Informe sobre la implementación de la Convención sobre los Derechos de las Personas con Discapacidad ${ }^{24}$, Colombia reporta que se encuentra implementando la Ley 1618 del 2013, donde se da un plazo máximo de diez años para garantizar el acceso de las personas con discapacidad al transporte público y ocho años para adecuar en términos de accesibilidad las vías, aeropuertos y terminales del país. Lo cual demuestra que se toman medidas a largo plazo, cuando se necesitan acciones inmediatas para el mejoramiento de la calidad de vida de las personas con discapacidad. El logro de la visibilidad social de los problemas de movilidad de las personas con discapacidad ayudará no solo a que se tomen las medidas necesarias para solucionar las falencias presentes, sino que al mismo tiempo posibilitará que la persona con discapacidad perciba su entorno como más justo e incluyente, mejorando el aspecto subjetivo de su calidad de vida ${ }^{52}$. Contrario a la situación que se generaría en ambientes hostiles estructuralmente a su presencia, en los cuales "las desventajas, la segregación y la inaccesibilidad a recursos no están determinadas por la deficiencia orgánica que puede presentar una persona, sino que obedecen a las consecuencias de tener que afrontar un entorno que no está diseñado para la diversidad"53. Po tanto, los profesionales de las distintas áreas, deben intervenir en el proceso de proposición de nuevas Leyes a nivel nacional que busquen disminuir el impacto psicosocial de las discapacidades ${ }^{54}$.

Terminando este recorrido histórico encaminado a conocer la temática de la discapacidad desde el ámbito legislativo, es importante resaltar que se ha trabajado por mejorar las condiciones de estas personas desde lo social y lo político. Es notable que la sociedad y los gobiernos han 
realizado esfuerzos por mejorar sus condiciones de vida, buscando verlos como personas iguales a las demás, aunque con sus elementos diferenciadores ${ }^{55}$; la pérdida permanente de alguna función física y los limitantes que esto acarrea han sido un factor presente en la experiencia humana. La existencia de la discapacidad depende de que sea considerada como tal, y en esa denominación se ve implicado el sistema de salud. Como se pudo notar, la legislación en salud en Colombia ve a la discapacidad como una enfermedad, partiendo desde una influencia social y cultural ${ }^{56}$.

\section{DISCUSIÓN}

En Colombia, 2.149.710 de personas presentan alguna discapacidad. Esta cifra fue reportada por el DANE (2012) ${ }^{3}$, la cual en ese entonces equivalía al 4,7 por ciento de los habitantes y ubica a Colombia como la segunda nación con mayor prevalencia del fenómeno en América Latina, después de Brasil. Sin embargo, en América Latina, ha sido tardío el establecimiento de medidas legislativas para que la población con discapacidad pueda gozar de sus derechos. En Argentina, por ejemplo, es en 1981, con la Ley 22431, que se crea el sistema de protección integral de los discapacitados; en Bolivia fue en 1995 con la Ley 1678: Ley de la Persona con discapacidad; en Brasil fue en 1989 con la Ley 7.853: sobre el Apoyo a las Personas Portadoras de deficiencia y sobre su Integración Social; en Perú fue en 1998 con la Ley 27050 de la persona con discapacidad, etc. ${ }^{57}$.

En cuanto a la salud, la población con discapacidad debe recurrir a tutelas para hacer valer sus derechos, por la negativa de las distintas entidades a prestar servicios de calidad, a causa de los altos costos que ello les representa ${ }^{22}$. Y en lo referente a la educación, no resulta menos alarmante el dato de la encuesta de calidad de vida del DANE, en el año $2012^{3}$, la cual señalaba que en el rango etario de los 18 a los 39 años, entre la población con discapacidad, tan solo el 9.7\% alcanza un nivel de educación superior. Sin oportunidades efectivas de educación la problemática de la población con discapacidad no hace más que aumentar, reduciendo las posibilidades de encontrar empleos dignos y oportunidades de desarrollo personal y profesional.

Lo anterior se ve confirmado por los datos aportados por el Consejo Nacional de Política Económica y Social ${ }^{7}$, según los cuales se ha señalado que la población con discapacidad tiene altos niveles de desempleo, y cuando lo tienen perciben que son remunerados por debajo del trabajador común. Esta es una realidad contraria a los esfuerzos que desde organismos internacionales como la OIT se han venido realizando con el fin de garantizar cada vez con mayor eficacia el acceso de la población discapacitada a trabajo digno y equitativo, que permita la realización de sus proyectos personales. Y aunque el Estado colombiano ha realizado esfuerzos considerables en materia legislativa queda aún mucho por hacer en esta área del acceso al trabajo digno y la implementación de todas sus leyes. Pues se percibe en las estadísticas una brecha entre el texto de la norma y la realidad. Para comprobar lo anterior, la Ley estatutaria 1618 de 2013 establece que se debe "garantizar y asegurar el ejercicio efectivo de los derechos de las personas con discapacidad, mediante la adopción de medidas de inclusión... eliminando toda forma de discriminación por razón de discapacidad", ${ }^{15}$. Por lo cual, se rige bajo los principios que protegen los derechos de las personas con discapacidad (dignidad humana, respeto, autonomía individual, independencia, igualdad, equidad, Justicia, inclusión, progresividad en la financiación, equiparación de oportunidades, protección, no discriminación, solidaridad, pluralismo, accesibilidad, diversidad, respeto, aceptación de las diferencias y participación de las Personas con Discapacidad).

Al afirmar la Ley que se "evaluará cada 4 años la eficacia de las acciones afirmativas, los ajustes razonables y la sanción a la discriminación, como mecanismos para garantizar el ejercicio efectivo de los derechos de las personas con discapacidad"15; establece un plazo de evaluación de tales disposiciones legales, de forma tal que hacia el 2017 se procederá a evaluar el cumplimiento de las disposiciones emitidas, así como a valorar las omisiones de los diferentes entes territoriales; es de esta forma como el cuerpo legislativo de una nación avanza en perfeccionamiento intrínseco y en eficacia ciudadana. De igual forma afirma que es responsabilidad de las entidades públicas del orden nacional, departamental, municipal, distrital y local, en el marco del Sistema Nacional de Discapacidad "asegurar que todas las políticas, planes y programas, garanticen el ejercicio total y efectivo de sus derechos $^{15}$. Entre los resultado obtenidos se encuentra la generación de instituciones veedoras de la garantía de derechos conformada por las personas con discapacidad; a su vez, la vinculación de las mismas en procesos de gestión social para la población objetivo. De igual forma, en cada departamento se han creado comités departamentales/ municipales/locales de discapacidad con el objetivo de verificar el cumplimiento de la norma y exigir que sean respetados los derechos de las personas con discapacidad. 
Es deber del legislador emitir la Ley, pero es deber del Estado en su faceta judicial velar por el cumplimiento de dichas Leyes, así como por la eficaz aplicación de los distintos convenios que la nación firme con otros países o con entidades internacionales como la ONU, la cual en el 2006 instituyó la Convención sobre los Derechos de las Personas con Discapacidad, con el expreso propósito de: "promover, proteger y asegurar el goce pleno y en condiciones de igualdad de todos los derechos humanos y libertades fundamentales por todas las personas con discapacidad, y promover el respeto de sus dignidad inherente" 58 . Como parte de los deberes adquiridos por Colombia en esta Convención aparecen, entre otros:

- Implementar medidas legislativas y administrativas que hagan efectivos los derechos reconocidos en dicha convención.

- Eliminar o derogar de la legislación vigente todo reglamento o práctica discriminatoria hacia las personas con discapacidad.

- En los programas sociales y políticas públicas incluir la protección y promoción de los derechos de las personas con discapacidad

La legislación en Colombia es abundante, pero los formuladores de políticas no se encuentran bien informados sobre la realidad de la vida de las personas con discapacidad. Lo cual ha generado que el direccionamiento de los recursos y acciones del estado no sean efectivos para lograr el bienestar de esta población, llevando a las instituciones y sectores que brindan servicios a las personas con discapacidad a no efectuar un buen manejo de sus servicios ${ }^{59,60}$.

De la presente revisión se concluye que se han tomado medidas legislativas que favorecen a la población con discapacidad y que se ha incrementado la norma para proteger sus derechos; sin embargo, una de las principales dificultades que se encuentran es el desconocimiento de la legislación, la difusión de la legislación y el empoderamiento de las personas con discapacidad para ejercer sus derechos respaldados por la legislación. De igual forma, es importante la inclusión de estos temas en la formación profesional ya que contribuye al desarrollo académico e investigativo de una necesidad social que incrementa con el correr de los años y que implica no solo el conocimiento, la sensibilización sino también la innovación y gestión del cambio de cultura y de gestión política. Aunque el camino recorrido ha sido importante, aún queda mucho por hacer en materia legislativa y de control, en pro de dar plenas garantías a la población discapacitada sobre el ejercicio de sus derechos.

\section{REFERENCIAS BIBLIOGRÁFICAS}

1. Organización Mundial de la Salud (OMS). 10 datos sobre la discapacidad [internet]. [Consultado 2014 Febrero 15]. Disponible en: http://www.who.int/mediacentre/ factsheets/fs352/es/

2. Angarita M. Políticas y conceptos en discapacidad: Un binomio por explorar. [Tesis Doctoral]. Bogotá: Universidad Nacional de Colombia; 2007.

3. Departamento Administrativo Nacional de Estadística (DANE). Encuesta Nacional de Calidad de Vida 2012 [internet]. [Consultado 2014 Marzo 26]. Disponible en: http://www.dane.gov.co/index.php/estadisticassociales/calidad-de-vida-ecv

4. Sistema Integral de Información de la Protección Social (SISPRO). Registro de localización y caracterización de personas con discapacidad [internet]. [Consultado 2014 Febrero 15]. Disponible en: http://www.sispro.gov.co/

5. Abberley P. The Concept of Oppression and the Development of a Social Theory of Disability. Disability, Handicap and Society. 1987; 2 (1): 5-19.

6. Discapacidad Colombia. Legislación [Sitio en Internet]. [Consultado 2014 Febrero 15]. Disponible en: http:// discapacidadcolombia.com/modules.php?name $=$ Cont ent\&pa = showpage\&pid $=34$

7. Consejo Nacional de Política Economía y Social (CONPES). Política Pública Nacional de Discapacidad e Inclusión Social [internet]. [Consultado 2014 Febrero 15]. Disponible en: http://www.colombiaaprende.edu. co/html/micrositios/1752/articles-335918_archivo_pdf. pdf

8. Colombia. Ministerio de Salud. Resolución 14861 de 1985 Por la cual se dictan normas para la protección, seguridad, salud y bienestar de las personas en el ambiente y en especial de los minusválidos. Bogotá: Ministerio de Salud; 1985

9. Organización de las Naciones Unidas (ONU). Las Naciones Unidas y las Personas con Discapacidad - Los primeros cincuenta años [internet]. [Consultado 2014 Febrero 20]. Disponible en: http://www.un.org/spanish/ esa/social/disabled/dis50y10.htm

10. Detrich R, Keyworth R, States J. Advances in evidencebased education: A road map to evidence based education. 2008. Oakland, CA: The Wing Institute.

11. Colombia. El Congreso de Colombia. Ley 12 de 1987 por la cual se suprimen algunas barreras arquitectónicas y se dictan otras disposiciones. Diario Oficial, 37765 (Enero 27 de 1987).

12. Colombia. Ministerio de Trabajo y Seguridad Social. Ley 82 de 1988 Por medio de la cual se aprueba el Convenio 159 sobre la readaptación profesional y el empleo de personas inválidas, adoptado por la Conferencia General de la Organización Internacional del Trabajo en su 69a. reunión, Ginebra, 1983. Diario Oficial, 38626 (Diciembre 23 de 1988). 
13. Colombia. Ministerio de Transporte. Ley 769 de 2002 Por la cual se expide el Código Nacional de Tránsito Terrestre y se dictan otras disposiciones. Diario Oficial, 44932 (Septiembre 13 de 2002).

14. Colombia. Ministerio de la Protección Social. Ley 1145 de 2007 por medio de la cual se organiza el Sistema Nacional de Discapacidad y se dictan otras disposiciones. Diario Oficial, 46685 (Julio 10 de 2007).

15. Congreso de Colombia. Ministerio de Salud y Protección Social. Ley Estatutaria 1618 de 2013 por medio de la cual se establecen las disposiciones para garantizar el pleno ejercicio de los derechos de las personas con discapacidad. Bogotá: Ministerio de Salud y Protección Social; 2013.

16. Nullvalue. Qué es una ley estatutaria [internet]. [Consultado 2014 Marzo 10]. Disponible en: http:// www.eltiempo.com/archivo/documento/MAM-138592

17. Organización Mundial de la Salud (OMS). Informe Mundial Sobre la Discapacidad [internet]. [Consultado 2014 Marzo 24]. Disponible en: http://www.who.int/ disabilities/world_report/2011/summary_es.pdf

18. Colombia. Ministerio de Hacienda y Crédito Público. Ley 60 de 1993 por la cual se dictan normas orgánicas sobre la distribución de competencias de conformidad con los artículos 151 y 288 de la Constitución Política y se distribuyen recursos según los artículos 356 y 357 de la Constitución Política y se dictan otras disposiciones. Diario Oficial, 40987 (Agosto 12 de 1993).

19. Colombia. Congreso de Colombia. Ley 100 de 1993 Por la cual se crea el sistema de seguridad social integral y se dictan otras disposiciones. . Diario Oficial, 41148 (Diciembre 23 de 1993).

20. Carrasquilla G, Martínez S, Latorre M, García S, Rincón C, Pardo C, et al. La discapacidad en el contexto del Sistema General de Seguridad Social en Salud en Colombia: Lineamientos, Epidemiología, e Impacto Económico [internet]. [Consultado 2014 Marzo 10]. Disponible en: http://mesadesplazamientoydiscapacidad.files. wordpress.com/2010/05/doc-fundac-2009.pdf

21. Organización de las Naciones Unidas (ONU). Convención sobre los derechos de las personas con discapacidad [internet]. [Consultado 2014 Marzo 10]. Disponible en: https://www.un.org/spanish/disabilities/default. asp?id $=497$

22. Correa ML. Panorama de la protección jurisprudencial a los derechos humanos de las personas con discapacidad en Colombia. Universitas. 2009; 118: 115-139.

23. Hernández JJ, Hernández UI. Una aproximación a los costos indirectos de la discapacidad en Colombia. Revista de Salud Pública .2009; 7(2): 130-144.

24. República de Colombia. Informe inicial sobre la implementación de la Convención sobre los Derechos de la Personas con Discapacidad en Colombia. Bogotá: 2013.
25. Colombia. Ministerio de la Protección Social. Ley 1438 de 2011 Por medio de la cual se reforma el Sistema General de Seguridad Social en Salud y se dictan otras disposiciones. Diario Oficial, 47957 (Enero 19 de 2011).

26. Trujillo A. Psicología de la discapacidad [internet]. [Consultado 2014 Marzo 12]. Disponible en: http://www. discapacidadonline.com/wp-content/uploads/2012/10/ psicologia-de-la-discapacidad.pdf

27. Cobo ME, Sandoval CC, Alvarado RY. Magnitud de la discapacidad en Boyacá, Colombia. Revista Salud Pública. 2012; 14(5): 776-788.

28. Colombia. Constitución Política de 1991 [internet]. [Consultado 2014 Marzo 12]. Disponible en: http:// www.alcaldiabogota.gov.co/sisjur/normas/Norma1. jsp? $\mathrm{i}=4125$

29. Díaz OC. Concepción de la atención educativa de personas con discapacidad. Documento de trabajo. Bogotá: Ministerio de Educación Nacional. 2003.

30. Boluarte A, Méndez J, Martell R. Programa de entrenamiento en habilidades sociales con retraso mental leve y moderado. Mosaico Científico. 2006; 3(1): 34-42.

31. Parra C. Borradores de investigación. Población con discapacidad en Colombia. Aspectos sociales, económicos y políticos. Bogotá: Facultad de Jurisprudencia Universidad del Rosario. 2003.

32. Gutiérrez B, Prieto M. Manual de evaluación y entrenamiento en habilidades sociales para personas con retraso mental [internet]. [Consultado 2014 Mayo 10]. Disponible en: http://www.feapsmurcia. org/feaps/FeapsDocumentos.NSF/08db27d07184be5 0c125746400284778/b72f09f53bf65706c12578d30046d 6f0/\$FILE/Presentaci\%C3\%B3n.pdf

33. Sánchez P. Psicología educativa de hoy. Reflexiones sobre el papel del psicólogo educativo en el proceso educativo de cara al nuevo siglo. . En M. Brea, E. Rodríguez, \& M. Alonso (Eds.), 30 años de la psicología dominicana: Pasado, presente y futuro. Santo Domingo: Fundación Cátedra Iberoamericana; 1998. 94-106.

34. Arvilla A, Palacio L, Arango C. El psicólogo educativo y su quehacer en la institución educativa. Revista de la Facultad de Ciencias de la Salud. 2011; 8(2): 258-261.

35. Arena B, Jaramillo J, Marmolejo P, Cruz C. Calidad de vida e inclusión laboral de personas con discapacidad. Cali 2008.2011. Ciencia \& Salud. 2013; 1(3): 39-47.

36. Cruz MM, García A. La Economía y la Psicología ante el problema de la inactividad de las personas con discapacidad. Revista de Fomento Social. 2011; 66: 251 -284

37. García SC, Malo M. "Discapacidad y mercado de trabajo en la Unión Europea”. Cuadernos Aragoneses de Economía. 2002; 12(2): 237-255.

38. Gannon B, Nolan B. Disability and Labour Market Participation in Ireland. Economic and Social Review. 2004; 35(2): 135-155. 
39. Borda A, Valera M, Cruz N. Vinculación de discapacitados en las organizaciones [internet]. [Consultado 2014 Mayo 12]. Disponible en: http://intellectum.unisabana.edu. co:8080/jspui/handle/10818/4527

40. Colombia. Ministerio de Trabajo y Seguridad Social. Ley 361 de 1997 por la cual se establecen mecanismos de integración social de la personas con limitación y se dictan otras disposiciones. Diario Oficial, 42978 (Febrero 11 de 1997).

41. Cardona D, Agudelo A, Restrepo L, Segura A. Educación, salud y empleo de la población con algún tipo de limitación. Medellín, 2008. Hacia la promoción de la salud. 2012; 17(1): 77-91.

42. Colella $A$, Bruyère $S$. Disability and employment: New directions for industrial and organizational psychology. 2011. En S. Zedeck (Ed. de la serie), APA handbook of industrial and organizational psychology: Volum 1 Building and developinig the organization (pp. 473-503). Washington, D.C.: American Psychological Association.

43. Pérez V, Alcóver C. Apoyo social, satisfacción laboral y abandono en trabajadores con discapacidad. Boletín de Psicología. 2011; 102: 23-41.

44. Schur L, Kruse D, Blanck P. Corporate culture and the employment of persons with disabilities. Behavioral Science and the Law. 2005; 23: 3-20.

45. Zondek A, Zepeda M, González F. Recabarren E. Discapacidad en Chile: pasos hacia un modelo integral de funcionamiento humano. Fondo Nacional de la Discapacidad. Santiago de Chile: Maval Ltda; 2006.

46. Colombia. Ministerio de Trabajo y Seguridad Social. Ley 12 de 1987 por la cual se suprimen algunas barreras arquitectónicas y se dictan otras disposiciones. Diario Oficial, 37765 (Enero 27 de 1987).

47. Colombia. Ministerio de Transporte. Decreto 1660 de 2003 por el cual se reglamenta la accesibilidad a los modos de transporte de la población en general y en especial de las personas con discapacidad. Diario Oficial, 45222 (Junio 18 de 2003).

48. Colombia. Ministerio de Ambiente, Vivienda y Desarrollo Territorial. Decreto 1538 de 2005 por el cual se reglamenta parcialmente la Ley 361 de 1997. Diario Oficial, 45913 (Mayo 19 de 2005).

49. Colombia. Ministerio de Transporte. Ley 1083 de 2006 por medio de la cual se establecen algunas normas sobre planeación urbana sostenible y se dictan otras disposiciones. Diario Oficial, 46346 (Julio 31 de 2006).

50. Colombia. Ministerio de Transporte. Resolución 4659 de 2008 por la cual se adoptan unas medidas en materia de accesibilidad a los sistemas de transporte público masivo municipal, distrital y metropolitano de pasajeros. Diario Oficial, 47169 (Noviembre 10 de 2008).

51. Colombia. Ministerio de Transporte. Ley 1287 de 2009 Por la cual se adiciona la Ley 361 de 1997. Diario Oficial, 47280 (Marzo 3 de 2009).

52. Muyor J. Intervenir en la vida de otros: Trabajo social, discapacidad y derechos humanos. Trabajo Social Difusión. 2011; 87: 17-22.

53. Alfaro RL. Psicología y discapacidad: Un encuentro desde el paradigma social. Revista Costarricense de Psicología. 2013; 32(1): 63-74.

54. Strauss AL. Sales A. Bridging the gap between disability studies and disability services in higher education: A model center on disability. Journal of Postsecondary Education and Disability. 2010; 23(1): 79-84.

55. Juárez F, Holguín E, Salamanca A. Aceptación o rechazo: perspectiva histórica sobre la discapacidad, la rehabilitación y la psicología de la rehabilitación. Psicología y Salud. 2006; 16(2): 187.197.

56. López JM. Breve historia de la medicina. Madrid: Alianza Editorial; 2000

57. Programa de Naciones Unidas para el Desarrollo PNUD. Compendio de legislación sobre discapacidad [internet]. [Consultado 2014 Abril 20]. Disponible en: http:// conadis.salud.gob.mx/descargas/pdf/CLSDtomo1.pdf

58. Organización Internacional de la Naciones Unidas. Convención sobre los derechos de las personas con discapacidad [internet]. [Consultado 2014 Febrero 20]. Disponible en: www.un.org/esa/socdev/enable/ documents/tccconvs.pdf

59. Gómez C, Cuervo C. Conceptualización de discapacidad: reflexiones para Colombia [Tesis de Maestría en Discapacidad e Inclusión Social]. Bogotá: Universidad Nacional de Colombia, Facultad de Medicina; 2007.

60. Cordeu C. Reflexiones en torno a la inclusión sociocomunitaria de personas con discapacidad intelectual: escuchando la voz de sus protagonistas. [Tesis de Maestría en Psicología]. Santiago de Chile: Universidad de Chile. Facultad de Ciencias Sociales; 2008.

Para citar este artículo: Martínez-Rozo A, Uribe-Rodríguez A, Velázquez-González H. La discapacidad y su estado actual en la legislación colombiana. Duazary. 2015 jun; 12 (1): $49-58$ 